This item was submitted to Loughborough's Research Repository by the author.

Items in Figshare are protected by copyright, with all rights reserved, unless otherwise indicated.

\title{
Shedding light on topological superconductors
}

PLEASE CITE THE PUBLISHED VERSION

https://doi.org/10.1103/PhysRevB.98.064502

\section{PUBLISHER}

(C) American Physical Society

VERSION

AM (Accepted Manuscript)

\section{PUBLISHER STATEMENT}

This work is made available according to the conditions of the Creative Commons Attribution-NonCommercialNoDerivatives 4.0 International (CC BY-NC-ND 4.0) licence. Full details of this licence are available at: https://creativecommons.org/licenses/by-nc-nd/4.0/

\section{LICENCE}

CC BY-NC-ND 4.0

\section{REPOSITORY RECORD}

Villegas, K.H.A., V.M. Kovalev, Feodor Kusmartsev, and I.G. Savenko. 2019. "Shedding Light on Topological Superconductors". figshare. https://hdl.handle.net/2134/34503. 


\title{
Shedding light on topological superconductors
}

\author{
K. H. A. Villegas, ${ }^{1}$ V. M. Kovalev,${ }^{2,3}$ F. V. Kusmartsev, ${ }^{4,5}$ and I. G. Savenko ${ }^{1,6}$ \\ ${ }^{1}$ Center for Theoretical Physics of Complex Systems, \\ Institute for Basic Science (IBS), Daejeon 34126, Korea \\ ${ }^{2}$ A.V. Rzhanov Institute of Semiconductor Physics, \\ Siberian Branch of Russian Academy of Sciences, Novosibirsk 630090, Russia \\ ${ }^{3}$ Department of Applied and Theoretical Physics, \\ Novosibirsk State Technical University, Novosibirsk 630073, Russia \\ ${ }^{4}$ Department of Physics, Loughborough University, Loughborough LE11 3TU, United Kingdom \\ ${ }^{5}$ Micro/Nano Fabrication Laboratory Microsystem and THz Research Center, Chengdu, Sichuan, China \\ ${ }^{6}$ Basic Science Program, Korea University of Science and Technology (UST), Daejeon 34113, Korea
}

(Dated: July 7, 2018)

\begin{abstract}
We propose an optical approach to monitor superconductors in conjunction with a normal metal layer. Effectively such a hybrid system represents a resonator, where electrons are strongly coupled with light. We show that the interaction of light with the superconductor is strongly boosted in the presence of the neighboring metal and as a result, the electromagnetic power absorption of the system is dramatically enhanced. It manifests itself in a giant Fanolike resonance which can uniquely characterize the elementary excitations of the system. Our approach is especially promising for topological superconductors, where Majorana fermions could be revealed and controlled by light.
\end{abstract}

\section{INTRODUCTION}

Superconductivity is conventionally considered to be a material property difficult to characterize with light, due to weak light-matter interaction in superconducting condensates. To test whether materials are superconductors, electric (resistivity-based) and magnetic (Meissner effect-based) techniques are routinely used. However, optics would be very helpful if we want to monitor hybrids of such fascinating classes of materials as topological insulators (TI) [1-5] and Weyl semimetals (WM) [6-13]. In this framework, superconductors should be considered as candidates to reveal new topological properties, thus fostering a revisit of existing experimental techniques.

Topological superconductors behaving metallic on the surface and superconducting in the bulk naturally combine the properties of both metal and superconductor, which is the key problem for their discovery. On one hand, electrical conductivity measurements used to study conventional superconductors have proved to be challenging due to a mutual influence of the free electrons associated with the metallic surface and the Cooper pairs of the superconducting bulk. On the other hand, diamagnetic Meissner magnetization measurements require a minimal volume, which is an issue for surface topological superconductivity.

In this article, we propose an optical approach to monitor behavior of superconductors. As an important ingredient, it requires coupling of the superconductor to a metallic layer with plasmonic gapless excitations. Their hybridization with flat bands and superconducting excitations leads to a giant light absorption due to a high density of states. As a result, a film of topological material deposited on the thin metallic layer will have hybrid elementary excitations. The significant result that we demonstrate below is that these excitations are highly optically active; they display strong resonances in opti- cal absorption measurements, thereby characterizing the material under study.

Various hybrid normal metal-superconductor or semiconductor-superconductor systems, in which a twodimensional electron gas (2DEG) is in contact with Cooper pairs, have been broadly considered in literature. Examples of the widespread implementation of such hybrid systems include a Josephson junction or a Josephson tunnel junction [15] aimed at cooling (as a heat sink), observation of Majorana fermions [16, 17] and zero modes [18, 19], and an enhancement of the degree of photon pair entanglement [20].

Further, recent studies show that there are advantages in using optical scattering in plasmons especially in the context of plasmon-based refractive index sensing. For example in graphene, it was shown that such a plasmonic sensor has a high surface sensitivity[21] and further, the light scattering can be controlled by dc current [22]. This was used to study non-local response and presence of adatoms and impurities[24, 25].

Recently, it was shown that hybrid systems also allow new mechanisms of superconductivity itself using interaction with excitons [26] or exciton polaritons [27, 28] in semiconductor structures, where the latter can serve as an auxiliary to increase $T_{c}$. From an application-oriented perspective, semiconductor-based hybrid structures can be employed in such devices as tunnel diodes [29] and optoelectronic circuits for high-bandwidth information processing [30, 31]. Furthermore, the most recent advances in molecular beam epitaxial heterostructure growth techniques suggest a route to create high-quality hybrid structures [32].

We show here that a metallic layer located in the vicinity of a superconductor can dramatically enhance lightsuperconductor coupling, such that the superconducting properties can ultimately be well characterized by the absorption spectrum of the hybrid system. Specifically, 

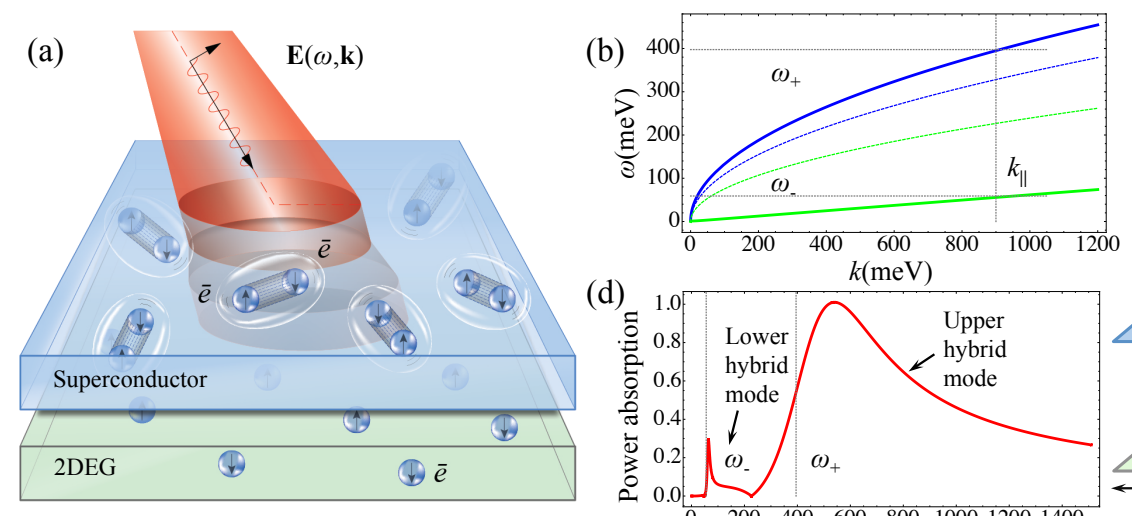

(c)
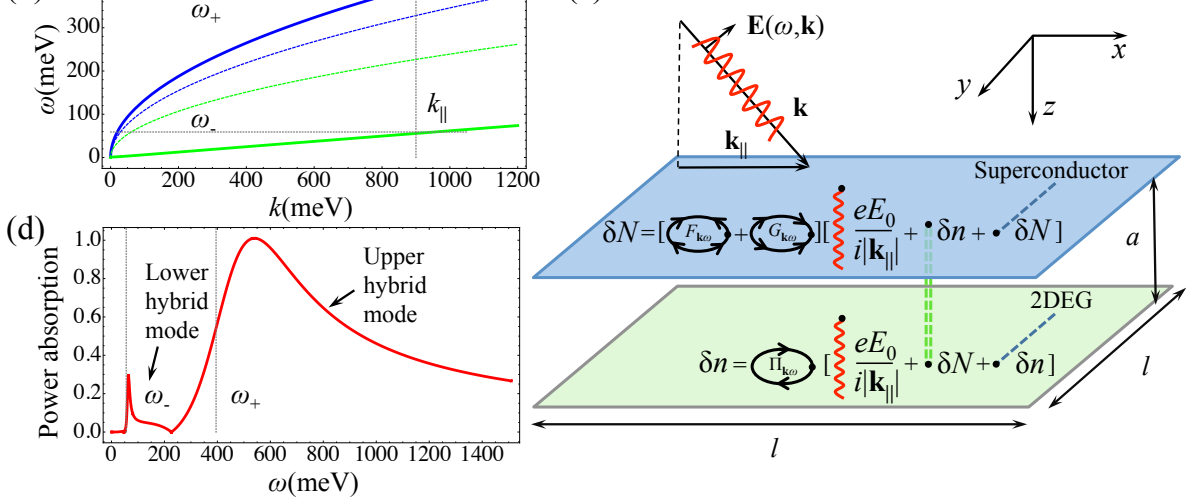

FIG. 1. System schematic. (a) Hybrid normal metal-superconductor structure exposed to an electromagnetic field of incident light. (b) Dispersions of hybrid eigenmodes of the system: $\omega$ as a function of $k$ for $m_{N}=m_{S}=1$ (green and blue solid curves). The dashed curves of the corresponding colors show the individual modes of each layer when the interlayer interaction is switched off. (c) Schematic of in-layer, inter-layer and light-matter interaction in the system manifesting itself in fluctuations of electron and Cooper pair densities, $\delta n$ and $\delta N$, and polarization operators $F_{\mathbf{k} \omega}, G_{\mathbf{k} \omega}$, and $\Pi_{\mathbf{k} \omega}$. (d) Spectrum of electromagnetic power absorption demonstrating the Fano resonance profile.

we demonstrate that the system reveals a giant hybrid Fano resonance [33, 34], which arises in both normal and superconducting hybrid subsystems due to their mutual influence. The shape and positions of the peaks (and the dip) of the Fano resonance may uniquely characterize both the superconducting and metallic subsystems, especially the value of the superconducting gap and therefore the order parameter, its symmetry and critical temperature. Thus, our findings open a prospective method, being optical and noninvasive, for the characterization and testing of materials for superconductivity.

\section{SYSTEM SCHEMATIC AND HYBRID EIGENMODES}

Let us consider a system with two parallel layers of a normal metal and a superconductor, as illustrated in Fig. 1a. The two layers are assumed to be sufficiently well separated $\left(10^{-6}-10^{-5} \mathrm{~cm}\right)$ so that tunneling and proximity effects can be ignored. The electrons in the normal metal interact via Coulomb interaction, which has the Fourier image given by $v_{k}=2 \pi e^{2} / k$, where $\mathbf{k}$ is in-plane momentum (lying in the $x y$ plane). The electrons between the two layers are also Coulomb-coupled, and the Fourier image of the interlayer interaction reads $u_{k}=2 \pi e^{2} \exp (-a k) / k$, where $a$ is the separation between the layers. The electromagnetic wave is polarized along the $x$-axis, $\mathbf{E}(\mathbf{r}, t)=\hat{\mathbf{x}} E_{0} e^{-i\left(k_{\perp} z+\mathbf{k}_{\|} \cdot \mathbf{r}+\omega t\right)}$ where $\mathbf{k}_{\|}, \omega$, and $\mathbf{r}$ are the in-plane wave vector of the field, frequency, and coordinate, respectively.

The polarization function of a (topologically trivial) superconductor at zero temperature can be presented in form [36]

$$
P_{\mathbf{k} \omega}=\sum_{\mathbf{p}} \frac{E_{\mathbf{p}} E_{\mathbf{p}+\mathbf{k}}-\xi_{\mathbf{p}} \xi_{\mathbf{p}+\mathbf{k}}-\Delta^{2}}{E_{\mathbf{p}} E_{\mathbf{p}+\mathbf{k}}}\left(\frac{1}{\omega+i \delta-E_{\mathbf{p}}-E_{\mathbf{p}+\mathbf{k}}}-\frac{1}{\omega+i \delta+E_{\mathbf{p}}+E_{\mathbf{p}+\mathbf{k}}}\right)=G_{\mathbf{k} \omega}+F_{\mathbf{k} \omega},
$$

where $2 \Delta$ is the superconducting gap, $\xi_{\mathrm{p}}$ is the single-particle energy of the electron measured with respect to the chemical potential, $\mu$, and $E_{\mathbf{p}} \equiv \sqrt{\xi_{\mathbf{p}}^{2}+\Delta^{2}}$ is the quasiparticle excitation energy. The sum in (1) can be converted into an integral, which can be evaluated analytically (see Appendix A). This gives us the real and imaginary parts of the polarization operator $P_{k \omega}$ (we will use $P_{k \omega}$ instead of $P_{\mathbf{k} \omega}$ in what follows):

$$
\begin{aligned}
P_{k \omega}^{R} & =\frac{p_{S F}^{2} k^{2}}{\pi m_{S}} \cdot \frac{1}{\omega^{2}-4 \Delta^{2}}, \\
P_{k \omega}^{I} & = \begin{cases}0, & 0 \leq \omega \leq 2 \Delta \\
-\frac{p_{S F}^{2} k^{2}}{2 m_{S} \omega} \cdot \frac{1}{\sqrt{\omega^{2}-4 \Delta^{2}}}, & 2 \Delta<\omega\end{cases}
\end{aligned}
$$


where $p_{S F}$ is the Fermi momentum and $m_{S}$ is effective electron mass in the superconductor.

The calculation of the electron gas polarization is standard [37] and here we simply provide the result:

$$
\begin{aligned}
& \Pi_{k \omega}^{R}=\frac{p_{N F}^{2}}{2 \pi^{2} m_{N}} \cdot \frac{k^{2}}{\omega^{2}}, \\
& \Pi_{k \omega}^{I}= \begin{cases}-\frac{m_{N}}{\pi}\left[\sqrt{1-\left(\frac{m_{N} \omega}{p_{N F}}-\frac{k}{2 p_{N F}}\right)^{2}}-\sqrt{1-\left(\frac{m_{N} \omega}{p_{N F} k}+\frac{k}{2 p_{N F}}\right)^{2}}\right], & 0 \leq \omega<\frac{p_{N F}}{m_{N}} k-\frac{k^{2}}{2 m_{N}} \\
-\frac{m_{N}}{\pi} \sqrt{1-\left(\frac{m_{N} \omega}{p_{N F}}-\frac{k}{2 p_{N F}}\right)^{2}}, & \frac{p_{N F}}{m_{N}} k-\frac{k^{2}}{2 m_{N}}<\omega<\frac{p_{N F}}{m_{N}} k+\frac{k^{2}}{2 m_{N}} \\
0, & \frac{p_{N F}}{m_{N}} k+\frac{k^{2}}{2 m_{N}}<\omega,\end{cases}
\end{aligned}
$$

where $p_{N F}$ and $m_{N}$ are the Fermi momentum and electron effective mass in the normal metal layer, respectively.

Using the linear response theory, we can represent the electron density fluctuations in the normal layer, $\delta n_{k \omega}$, and Cooper pair density fluctuations in superconducting layer, $\delta N_{k \omega}$, as:

$$
\begin{aligned}
\delta n_{k \omega} & =\Pi_{k \omega}\left(v_{k} \delta n_{k \omega}+u_{k} \delta N_{k \omega}+W_{k \omega}^{(N)}\right), \\
\delta N_{k \omega} & =P_{k \omega}\left(v_{k} \delta N_{k \omega}+u_{k} \delta n_{k \omega}+W_{k \omega}^{(S)}\right),
\end{aligned}
$$

where $W_{k \omega}^{(N)}$ and $W_{k \omega}^{(S)}$ are the Fourier images of the potential energy caused by the external electric field (see Appendix B for details),

$$
W_{k \omega}^{(N)}=g_{N} \frac{e E_{0}}{i k}, \quad W_{k \omega}^{(S)}=g_{S} \frac{e E_{0}}{i k},
$$

where the couplings, $g_{N}=0,1$ and $g_{S}=0,1$, allow us to turn off the external field on either of the layers. In matrix form, Eq. (4) reads

$$
\left[\begin{array}{cc}
\Pi_{k \omega} v_{k}-1 & \Pi_{k \omega} u_{k} \\
P_{k \omega} u_{k} & P_{k \omega} v_{k}-1
\end{array}\right]\left[\begin{array}{c}
\delta n_{k \omega} \\
\delta N_{k \omega}
\end{array}\right]=-\left[\begin{array}{c}
\Pi_{k \omega} W_{k \omega}^{(N)} \\
P_{k \omega} W_{k \omega}^{(S)}
\end{array}\right] .
$$

The eigenmodes of the system can then be found by equating the determinant of the two-by-two matrix in (6) to zero, which yields:

$$
1-v_{k}\left(\Pi_{k \omega}+P_{k \omega}\right)+\left(v_{k}^{2}-u_{k}^{2}\right) \Pi_{k \omega} P_{k \omega}=0 .
$$

In particular, for the real part of this determinant we find:

$$
\begin{array}{r}
D_{k \omega}^{R} \equiv 1-v_{k}\left(\Pi_{k \omega}^{R}+P_{k \omega}^{R}\right)+\left(v_{k}^{2}-u_{k}^{2}\right) \Pi_{k \omega}^{R} P_{k \omega}^{R} \\
+\Pi_{k \omega}^{I} P_{k \omega}^{I}\left(u_{k}^{2}-v_{k}^{2}\right)=0 .
\end{array}
$$

Using Eqs. (2) and (3), we find that $\Pi_{k \omega}^{I}=0$ for $\omega>2 \Delta$ while $P_{k \omega}^{I}=0$ for $\omega<2 \Delta$. Hence the last term in Eq. (8) is zero for all positive $\omega$ and we yield

$$
1-v_{k}\left(\Pi_{k \omega}^{R}+P_{k \omega}^{R}\right)+\left(v_{k}^{2}-u_{k}^{2}\right) \Pi_{k \omega}^{R} P_{k \omega}^{R}=0 .
$$

Solving for $\omega$, we find two branches of dispersion of the hybrid modes:

$$
\begin{aligned}
\omega_{ \pm}^{2}(k)=2 \Delta^{2} & +\frac{e^{2} k}{2}\left(\frac{p_{N F}^{2}}{\pi m_{N}}+\frac{2 p_{S F}^{2}}{m_{S}}\right) \pm \\
& \pm \frac{1}{2} \sqrt{\xi_{-} \xi_{+}-4 \beta_{k}^{2}}, \quad \text { where } \\
\xi_{ \pm} & =\left[\left(2 \Delta \pm e p_{N F} \sqrt{\frac{k}{\pi m_{N}}}\right)^{2}+\frac{2 e^{2}}{m_{S}} p_{S F}^{2} k\right], \\
\beta_{k}^{2} & =\frac{2 e^{4} p_{N F}^{2} p_{S F}^{2}}{\pi m_{N} m_{S}} k^{2}\left(1-e^{-2 k a}\right) .
\end{aligned}
$$

Figure $1 \mathrm{~b}$ shows the hybrid modes for various $m_{N}=m_{S}$ (with bare modes presented for comparison). If the interlayer interaction is turned-off, by separating the layers sufficiently far apart, for example, the individual bare modes of the 2DEG and the superconductor has similar form as Eq. (10) except that we replace the last term inside the square root by $\beta_{k, \text { bare }}^{2} \equiv \frac{2 e^{4} p_{N F}^{2} p_{S F}^{2}}{\pi m_{N} m_{S}} k^{2}$.

\section{ELECTROMAGNETIC POWER ABSORPTION}

Further, the matrix equation (6) can be solved for the density fluctuations which yields:

$\delta n_{k \omega}=\frac{e E_{0}}{k} \frac{\left(N_{k \omega}^{R}+i N_{k \omega}^{I}\right) D_{k \omega}^{R}+\left(N_{k \omega}^{I}-i N_{k \omega}^{R}\right) D_{k \omega}^{I}}{\left(D_{k \omega}^{R}\right)^{2}+\left(D_{k \omega}^{I}\right)^{2}}$,

where $D_{k \omega}^{R}$ has already been given in Eq. (8) and

$$
\begin{aligned}
& N_{k \omega}^{R}=\left(g_{N} u_{k}-g_{S} v_{k}\right)\left(\Pi_{k \omega}^{I} P_{k \omega}^{R}+\Pi_{k \omega}^{R} P_{k \omega}^{I}\right)+g_{N} \Pi_{k \omega}^{I}, \\
& N_{k \omega}^{I}=-\left(g_{N} u_{k}-g_{S} v_{k}\right) \Pi_{k \omega}^{R} P_{k \omega}^{R}-g_{N} \Pi_{k \omega}^{R}, \quad(12) \\
& D_{k \omega}^{I}=\left(v_{k}^{2}-u_{k}^{2}\right)\left(P_{k \omega}^{I} \Pi_{k \omega}^{R}-P_{k \omega}^{R} \Pi_{k \omega}^{I}\right)-v_{k}\left(P_{k \omega}^{I}+\Pi_{k \omega}^{R}\right) .
\end{aligned}
$$

We are interested in the real part of (11) only, therefore we restrict ourselves to

$$
\delta n_{k \omega}=\frac{e E_{0}}{k} \frac{N_{k \omega}^{R} D_{k \omega}^{R}+N_{k \omega}^{I} D_{k \omega}^{I}}{\left(D_{k \omega}^{R}\right)^{2}+\left(D_{k \omega}^{I}\right)^{2}} .
$$

Under the assumption of linear response of the system, the Fourier component of the current in the 2DEG layer 
(a)
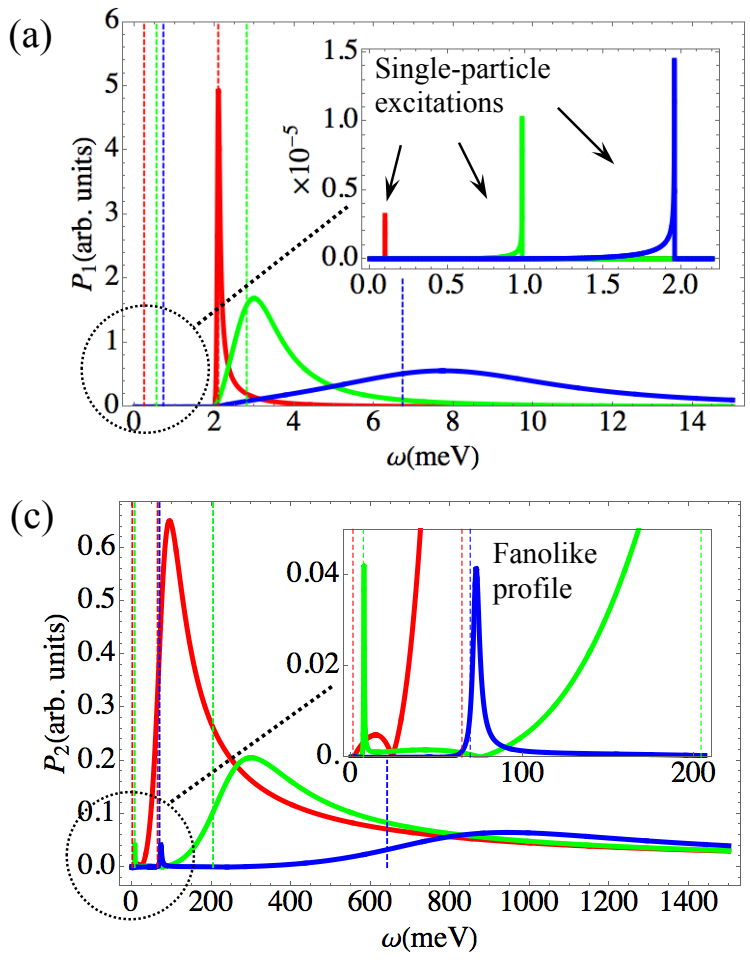

(b)

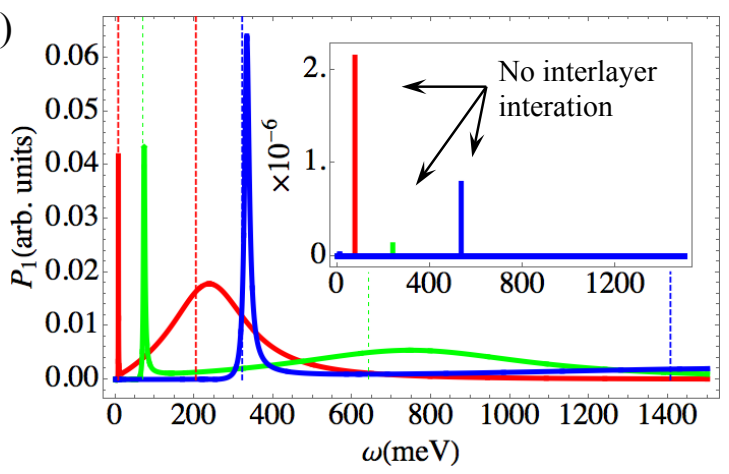

(d)

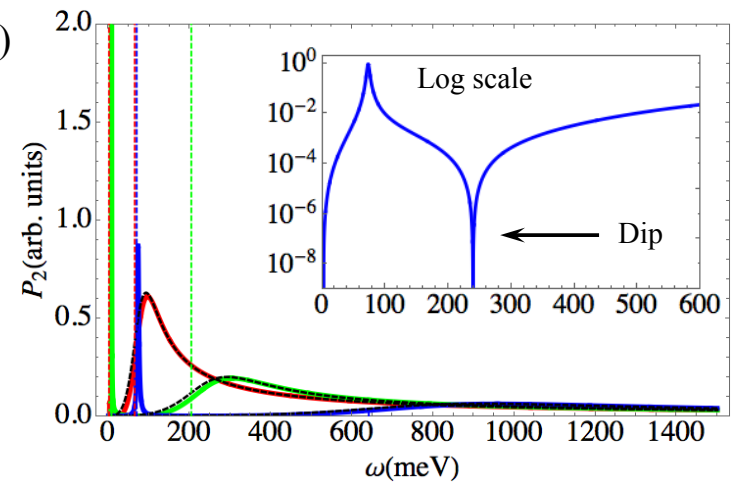

FIG. 2. Spectra. Power absorption monitored in 2DEG ((a) - (b)) and superconductor ((c)-(d)) as a function of $\omega$ for $\Delta=1.0$ $\mathrm{meV}$. Vertical dashed lines stand for the corresponding locations of the hybrid modes (within Eq. (10)). (a) $k=1.0 \times 10^{-3}$ (red curve), $1.0 \times 10^{-2}$ (green curve), and $1.0 \times 10^{-1} \mathrm{meV}$ (blue curve). Inset shows the range $0 \leq \omega<2 \Delta$, contributions to $P_{1}(\omega)$ due to single-particle excitations. To render these contributions visible, larger $k$ 's were used: $k=5.0 \times 10^{1}$ (red curve), $5.0 \times 10^{2}$ (green curve), and $1.0 \times 10^{3}$ (blue curve). (b) $k=1.0 \times 10^{1}$ (red curve), $1.0 \times 10^{2}$ (green curve), and $1.0 \times 10^{3} \mathrm{meV}$ (blue curve). Inset shows the case with no interlayer interaction. (c) Both layers are exposed to the EMF. Inset: zoom-in for small $\omega$ 's showing peaks caused by the lower hybrid modes. (d) No external field on the normal layer. Inset shows the log plot of the corresponding blue curve, manifesting the two peaks and the dip of the Fano resonance. In (c) and (d), $k=1.0 \times 10^{1}$ (red curves), $1.0 \times 10^{2}$ (green curves), $1.0 \times 10^{3} \mathrm{meV}$ (blue curves). Dashed black curves in (d) show the case when interlayer coupling is turned off.

can be written as

$$
J_{k^{\prime} \omega^{\prime}}=(2 \pi)^{3} \delta^{(2)}\left(\mathbf{k}^{\prime}-\mathbf{k}_{\|}\right) \delta\left(\omega^{\prime}-\omega\right) j_{k^{\prime} \omega} .
$$

The Dirac delta-functions in Eq. (14) explicitly implement the linear response assumption. Thus, both $\omega^{\prime}$ and $\mathbf{k}^{\prime}$ have specific values fixed by the external electromagnetic field (the factor $(2 \pi)^{3}$ is written for notational aesthetics). Since $\mathbf{E}(\mathbf{r}, t)$ and $\mathbf{J}(\mathbf{r}, t)$ have the same wave vector and frequency, the following formula can be used to compute the time-averaged power absorbed by the hybrid system as a function of frequency $\omega$ :

$$
\mathcal{P}(\omega)=\frac{1}{2}\left\langle\mathcal{R} e \int d^{2} r \mathbf{J}(\mathbf{r}, t) \cdot \mathbf{E}^{*}(\mathbf{r}, t)\right\rangle,
$$

where the integration is over the plane of the normal metal sample and $\langle\cdots\rangle$ denotes time-averaging. Normalizing Eq. (15) by $\int d^{2} r=L^{2}$, we obtain the specific power absorption coefficient (later simply referred to as power absorption):

$$
P_{1}(\omega) \equiv \frac{\mathcal{P}(\omega)}{L^{2}}=\frac{1}{2} \mathcal{R} e\left(j_{k \omega} E_{0}\right) .
$$

Utilizing the continuity equation $k j_{k, \omega}=-e \omega \delta n_{k, \omega}$ together with $\mathrm{Eq}(13)$, we find:

$$
\begin{aligned}
P_{1}(\omega) & =\frac{1}{2} \cdot \frac{e \omega}{k}\left|\mathcal{R} e\left(\delta n_{k, \omega}\right)\right| E_{0} \\
& =\frac{e^{2} \omega E_{0}^{2}}{2 k^{2}}\left|\frac{N_{k \omega}^{R} D_{k \omega}^{R}+N_{k \omega}^{I} D_{k \omega}^{I}}{\left(D_{k \omega}^{R}\right)^{2}+\left(D_{k \omega}^{I}\right)^{2}}\right| .
\end{aligned}
$$

We can follow a similar procedure to calculate the current of Cooper pairs in the superconductor (see also Appendix A). The power absorption then reads

$$
\begin{aligned}
P_{2}(\omega) & =\frac{1}{2} \cdot \frac{2 e \omega}{k}\left|\mathcal{R} e\left(\delta N_{k \omega}\right)\right| E_{0} \\
& =\frac{e^{2} \omega E_{0}^{2}}{k^{2}}\left|\frac{M_{k \omega}^{R} D_{k \omega}^{R}+M_{k \omega}^{I} D_{k \omega}^{I}}{\left(D_{k \omega}^{R}\right)^{2}+\left(D_{k \omega}^{I}\right)^{2}}\right|,
\end{aligned}
$$

where

$$
\begin{aligned}
& M_{k \omega}^{R}=\left(g_{N} u_{k}-g_{S} v_{k}\right)\left(P_{k \omega}^{R} \Pi_{k \omega}^{I}+P_{k \omega}^{I} \Pi_{k \omega}^{R}\right)+g_{S} P_{k \omega}^{I}, \\
& M_{k \omega}^{I}=\left(g_{S} v_{k}-g_{N} u_{k}\right) P_{k \omega}^{R} \Pi_{k \omega}^{R}-g_{S} P_{k \omega}^{R} .
\end{aligned}
$$

Formulas (17) and (18) account for the electron-electron and electron-Cooper pair interaction as well as the coupling of both the 2DEG and superconductor to light (see 
the schematic description of corresponding processes in Fig. 1c).

The resulting (schematic) power absorption by the hybrid system is presented in Fig. 1d, where due to the interplay of different interaction mechanisms, we expect to observe a Fano resonance. Let us consider the spectrum in detail.

\section{RESULTS AND DISCUSSION}

Figure 2 shows power absorption as a function of $\omega$ for different wave vectors $k$ when both the 2DEG and superconductor are exposed to the EMF. In Fig. 2a, the lower hybrid modes are below $2 \Delta$ and their contribution to the power absorption is suppressed, as can be seen by the lack of visible peaks in the vicinity of the three left-most dashed lines. The inset shows the contribution of the single-particle excitations. As can be seen, this contribution is negligible compared to the contribution of the hybrid modes (three peaks of the main plot). The locations of the peaks at higher frequencies nearly coincide with the corresponding dashed lines, showing that these peaks are primarily due to the upper hybrid modes. As $k$ increases, we observe a broadening (from red to blue curves).

Figure $2 \mathrm{~b}$ shows the power absorption for larger values of $k$. The lower hybrid modes now have significant contributions, as can be seen by the existence of three sharp peaks, since they are now located above the gap. For comparison, the inset shows the power absorption when the interlayer coupling is switched off. It shows a disappearance of the contribution from the upper hybrid modes primarily due to the superconductor. Comparison of the lower-mode contributions shows that the presence of the superconductor enhances the power absorption of the hybrid system by four orders of magnitude. Otherwise, the presence of the external EMF and the 2DEG does not dramatically influence the superconductor itself, and thus may serve as an auxiliary to monitor its behavior.

Figure 2c shows the power absorption spectrum for different $k$ 's when the EMF is exposed to both the 2DEG and superconductor. We observe a celebrated Fano resonance structure of the spectrum (see Inset). Figure 2d shows the power absorption when the external EMF at the normal layer is turned off. We immediately note that the contribution of the upper hybrid modes to the absorption spectrum is not significantly affected by switching off the field at the normal layer, as can be seen by comparing Figs. 2c and 2d. This behavior is quite expected. Surprisingly though, the contribution of the lower hybrid modes shows significant increase compared to the situation when the external field in the normal layer is switched on. Thus, we realize that the electronic layer must be very sensitive to the behavior of the superconductor, whereas the latter does not pay much attention to either the 2DEG or the light field. Indeed, switching

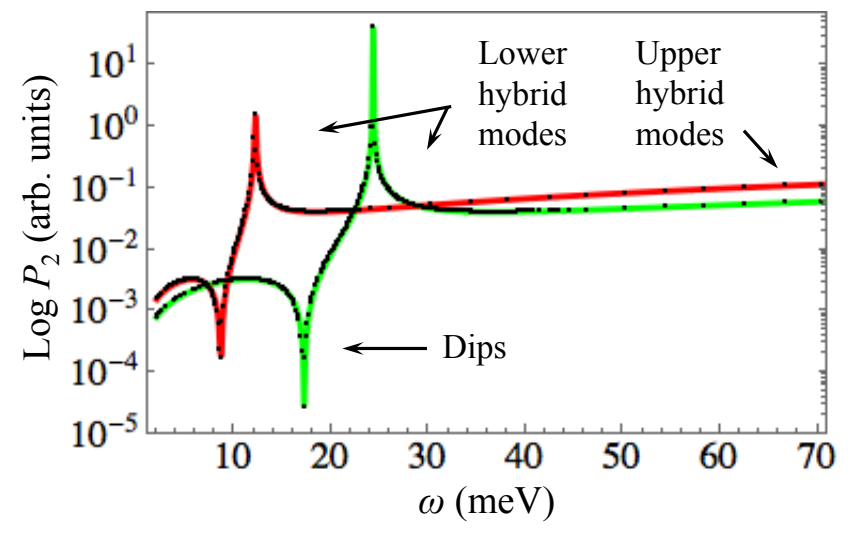

FIG. 3. Spectrum in topologically non-trivial case. Power absorption (in log scale) monitored in superconductor as a function of $\omega$ at $k=0.5 \times 10^{-1} \mathrm{meV}$ (red) and $k=1.0 \times$ $10^{-1} \mathrm{meV}$ (green). (Black dots show the results of numerical calculation.)

off the interlayer interaction removes the contribution of the lower modes, as expected (dashed black curves in Fig. 2d). We see that the second peak, which is mostly determined by the superconductor, remains nearly the same. Inset shows that in log scale, we observe both the two peaks of the Fanolike resonance and the dip.

Now let us consider the nontrivial topology (p-wave pairing). Then the superconducting gap reads $\Delta_{\mathbf{k}}=$ $f(k)\left(k_{x}-i k_{y}\right)=f(k) k e^{-i \phi}$, where $\phi$ is the polar angle measured with respect to the $k_{x}$ axis in $\mathrm{k}$-space and the function $f(k)$ depends on the concrete p-wave superconductor; it should be finite for all $k \in[0, \infty)$ and vanish as $k \rightarrow \infty$ (see the details in Appendix B). Dependence $\Delta_{\mathbf{k}}$ makes calculations more tricky but leads to qualitatively similar results. Figure 3 shows the resulting $P_{2}$ (compare with Fig. 2c, green curve). It should be noted, that here the Fanolike dip is shifted to the frequency range below the first peak.

\section{CONCLUSIONS AND OUTLINE}

We have studied the linear response of a hybrid twodimensional electron gas-superconductor system to an external electromagnetic field of light. Such systems have hybrid excitations that originate from gapless plasmons of the two-dimensional electron gas of the metallic layer and gapfull Bogolubov excitations of the bulk superconductor. We calculated these hybrid eigenmodes of the system and investigated the electromagnetic power absorption spectra. We found that these excitations exhibit a very strong coupling with the electromagnetic radiation, and showed that they display giant Fano resonances associated with a large light absorption. Such results therefore indicate a way to monitor the behavior of a superconductor exposed to light by measuring the spectrum of photoabsorption of the two-dimensional 
electron gas.

Herewith, we suggest a way to prepare samples of various hybrid-structured materials to test them for being superconductors via optics. That is, the proposed effect of the giant hybrid Fano resonance can be observed by measuring the optical response of two-layer metalsuperconductor systems composed of, for example, superconducting niobium film deposited on a thin metallic layer of copper, gold, silver, or tin. The proposed effect can be used to design various sensors and diagnostics of superconducting magnets with light.

Our result also opens the possibility of an alternative optical method for detecting Majorana fermions in topological superconductors, which is analogous to zerobias anomaly in tunneling spectroscopy[39-41]. Instead of tunneling, we propose to use light interaction to excite the electrons from the zero mode where a Majorana must be located. Chen et. al.[42] proposed a similar optical-detection method but using hybrid quantum dotnanomechanical resonator. In contrasts, in our method, the strong plasmon coupling, which produces a giant hybrid resonance, should provide a major advantage over the former.

Moreover, the recent discovery of high-temperature light-induced superconductivity in $\mathrm{K}_{2} \mathrm{C}_{60}$ [38] has stimulated an activity in the scientific community to test materials with light. Thus, our finding of enhanced light coupling in metal-superconducting hybrids alongside the possibility of testing is expected to open a new direction in this activity, since it creates many opportunities for discoveries of condensed states induced by light.

\section{ACKNOWLEDGEMENTS}

We thank Sergej Flach for useful discussions, Joel Rasmussen (RECON) for a critical reading of our manuscript, and Ekaterina Savenko for help with the figures. V. M. K. has been supported by the Russian Foundation for Basic Reaserch (Project No. 16-02-00565). K. H. V. and I. G. S. acknowledge the support of the Institute for Basic Science in Korea (Project No. IBSR024-D1).
[1] C. L. Kane, E. J. Mele, Phys. Rev. lett. 95, 146802 (2005).

[2] C. L. Kane, E. J. Mele, Phys. Rev. lett. 95, 226801 (2005).

[3] B. A. Bernevig, S. C. Zhang, Phys. Rev. lett. 96, 106802 (2006).

[4] L. Fu, C. L. Kane, Phys. Rev. B, 76, 045302 (2007).

[5] F. V. Kusmartsev, A. M. Tsvelik, JETP lett. 42, 257 (1985).

[6] D. T. Son, B. Z. Spivak, Physical Review B 88, 104412 (2013).

[7] B. Q. Lv, et al., Physical Review X 5, 031013 (2015).

[8] A. A. Soluyanov, D. Gresch, Z. Wang, Q. Wu, M. Troyer, X. Dai, B. A. Bernevig, Nature 527, 495 (2015).

[9] L. Yang, Z. Liu, Y. Sun, H. Peng, H. Yang, T. Zhang, B. Zhou, Y. Zhang, Y. Guo, M. Rahn, Nature Phys. 11, 728-732 (2015).

[10] S.-Y. Xu, N. Alidoust, I. Belopolski, Z. Yuan, G. Bian, T.-R. Chang, H. Zheng, V. N. Strocov, D. S. Sanchez, G. Chang, et al, Nature Phys. 11, 748-754 (2015).

[11] C.-K. Chiu, J. C. Teo, A. P. Schnyder, S. Ryu, Rev. Mod. Phys. 88, 035005 (2016).

[12] Y. Sun, S. C. Wu, M. N. Ali, C. Felser, B. Yan, Phys. Rev. B 92, 161107 (2015).

[13] C.-L. Zhang, S. Y. Xu, I. Belopolski, Z. Yuan, Z. Lin, B. Tong, G. Bian, N. Alidoust, C. C. Lee, S. M. Huang, et al., Nature Comm. 7, 10735 (2016).

[14] S. Borisenko, Q. Gibson, D. Evtushinsky, V. Zabolotnyy, B. Büchner, R. J. Cava, Phys. Rev. Lett. 113, 027603 (2014).

[15] T. Van Duzer and S. Kumar, Semiconductorsuperconductor hybrid electronics, Cryogenics 30, 1014 (1990).

[16] V. Mourik, K. Zuo, S. M. Frolov, S. R. Plissard, E. P. A. M. Bakkers, L. P. Kouwenhoven, Science 336, 1003
(2012).

[17] S. Takei, B. M. Fregoso, V. Galitski, and S. Das Sarma, Phys. Rev. B 87, 014504 (2013).

[18] S. Takei and V. Galitski, Phys. Rev. B 86, 054521 (2012).

[19] H. J. Suominen, M. Kjaergaard, A. R. Hamilton, J. Shabani, C. J. Palmstrom, C. M. Marcus, and F. Nichele, Phys. Rev. Lett. 119, 176805 (2017).

[20] M. Khoshnegar and A. H. Majedi, Phys. Rev. B 84, 104504 (2011).

[21] T. Wenger, G. Viola, J. Kinaret, M. Fogelstrom, and P. Tassin, 2D Mater. 4, 025103 (2017).

[22] T. Wenger, G. Viola, J. Kinaret, M. Fogelstrom, and P. Tassin, Phys. Rev. B 97, 085419 (2018).

[23] G. Viola, T. Wenger, J. Kinaret, and M. Fogelstrom, New J. Phys. 19, 073027 (2017).

[24] G. Viola, T. Wenger, J. Kinaret, and M. Fogelstrom, Phys. Rev. B 97, 085429 (2018).

[25] T. Wenger, G. Viola, and M. Fogelstrom, Phys. Rev. B 94, 205419 (2016).

[26] P. Skopelitis, E. D. Cherotchenko, A. V. Kavokin, and A. Posazhennikova, 120, 107001 (2018).

[27] F. P. Laussy, A. V. Kavokin, and I. A. Shelykh, Phys. Rev. Lett. 104, 106402 (2010).

[28] O. Cotlet, S. Zeytinoglu, M. Sigrist, E. Demler, and A. Imamoglu, Phys. Rev. B 93, 054510 (2016).

[29] A. Hayat, et al., Phys. Rev X 2, 041019 (2012).

[30] J. Shabani, et al., Phys. Rev. B 93, 155402 (2016).

[31] J. M. Shainline, S. M. Buckley, R. P. Mirin, and S. W. Nam, Phys. Rev. Appl. 7, 034013 (2017).

[32] R. Yan et al., Nature (London) 555, 183 (2018).

[33] A. E. Miroshnichenko, S. Flach, and Yu. S. Kivshar, Rev. Mod. Phys. 82, 2257 (2010).

[34] M. V. Boev, V. M. Kovalev, I. G. Savenko, Phys. Rev. B 94, 241408(R) (2016). 
[35] See Supplemental Material for the details of derivation of polarization operators and full summary of possible configurations of the system.

[36] P. I. Arseev, S. O. Loiko, N. K. Fedorov, Physics-Uspekhi 49 (1) 1 (2006).

[37] A. L. Fetter and J. D. Walecka, Quantum Theory of Many-Particle Systems, Dover Publications Inc. (2003).

[38] M. Mitrano et al., Nature 530, 461464 (2016).

[39] A. D. K. Finck, D. J. Van Harlingen, P. K. Mohseni, K. Jung, and X. Li, Phys. Rev. Lett. 110, 126406 (2013).
[40] E. J. H. Lee, X. Jiang, R. Aguado, G. Katsaros, C. M. Lieber, and S. De Franceschi, Phys. Rev. Lett. 109, 186802 (2012).

[41] S. Cho, R. Zhong, J. A. Schneeloch, G. Gu, and N. Mason, Sci. Rep. 6, 21767 (2016).

[42] H.-J. Chen, X.-W. Fang, C.-Z. Chen, Y. Li, and X.-D. Tang, Sci. Rep. 6, 36600 (2016).

\section{Appendix A: Evaluation of polarization functions of conventional and topological superconductors}

We consider the retarded polarization operator of the superconductor at zero temperature. Its general form, up to one-loop order is given by

$$
P_{k \omega}^{r e t}=\sum_{\mathbf{p}} \frac{E_{\mathbf{p}} E_{\mathbf{p}+\mathbf{k}}-\xi_{\mathbf{p}} \xi_{\mathbf{p}+\mathbf{k}}-\Delta_{\mathbf{p}}^{*} \Delta_{\mathbf{p}+\mathbf{k}}}{E_{\mathbf{p}} E_{\mathbf{p}+\mathbf{k}}}\left(\frac{1}{\omega+i \delta-E_{\mathbf{p}}-E_{\mathbf{p}+\mathbf{k}}}-\frac{1}{\omega+i \delta+E_{\mathbf{p}}+E_{\mathbf{p}+\mathbf{k}}}\right) .
$$

Let us evaluate the real, $P_{k \omega}^{R}$, and imaginary, $P_{k \omega}^{I}$, parts of this polarization operator $\left(P_{k \omega}^{r e t}=P_{k \omega}^{R}+i P_{k \omega}^{I}\right)$ separately.

\section{S-wave, Real part}

In the case of s-wave pairing, the gap is independent of the momentum. The real part then reads

$$
P_{k \omega}^{R}=\sum_{\mathbf{p}} \frac{E_{\mathbf{p}} E_{\mathbf{p}+\mathbf{k}}-\xi_{\mathbf{p}} \xi_{\mathbf{p}+\mathbf{k}}-\Delta^{2}}{E_{\mathbf{p}} E_{\mathbf{p}+\mathbf{k}}}\left(\frac{1}{\omega-E_{\mathbf{p}}-E_{\mathbf{p}+\mathbf{k}}}-\frac{1}{\omega+E_{\mathbf{p}}+E_{\mathbf{p}+\mathbf{k}}}\right) .
$$

First, we can convert the sum in (A2) into an integral by the replacement

$$
\sum_{\mathbf{p}} \rightarrow \frac{1}{(2 \pi)^{2}} \int d^{2} p=\frac{1}{(2 \pi)^{2}} \int_{0}^{\infty} d p p \int_{0}^{2 \pi} d \phi
$$

Without loss of generality, we choose $\mathbf{k}$ to point along the positive $x$ direction, so that $\phi$ is the angle between $\mathbf{p}$ and $\mathbf{k}$. We then perform the change of integration variable $\phi \rightarrow p_{1}$ :

$$
\begin{aligned}
p_{1}^{2} & \equiv|\mathbf{p}+\mathbf{k}|^{2}=p^{2}+2 k p \cos \phi+k^{2}, \\
d \phi & =-\frac{p_{1} d p_{1}}{p k \sin \phi},
\end{aligned}
$$

where

$$
\sin \phi= \pm \sqrt{1-\left(\frac{p_{1}^{2}-p^{2}-k^{2}}{2 p k}\right)^{2}}
$$

with the positive sign for $\phi \in[0, \pi]$ and negative sign for $\phi \in[\pi, 2 \pi]$. The integration over $\phi$ in Eq. (A3) becomes

$$
\int_{0}^{2 \pi} d \phi=\int_{0}^{\pi} d \phi+\int_{\pi}^{2 \pi} d \phi=4 \int_{p-k}^{p+k} \frac{p_{1} d p_{1}}{\sqrt{\left[(p+k)^{2}-p_{1}^{2}\right]\left[p_{1}^{2}-(p-k)^{2}\right]}} .
$$

Next, we can change the integration variables further:

$$
\begin{gathered}
p \rightarrow \xi_{p}=\frac{p^{2}}{2 m}-\mu, \\
d p=\sqrt{\frac{m}{2\left(\xi_{p}+\mu\right)}} d \xi_{p} .
\end{gathered}
$$


A similar change of variable is also done for $p_{1} \rightarrow \xi_{1}=\frac{p_{1}^{2}}{2 m}-\mu$. Eq. (A2) now turns into

$$
\begin{aligned}
P_{k \omega}^{R}= & \frac{m^{2}}{\pi^{2}} \int_{-\infty}^{\infty} d \xi_{p} \int_{\xi_{p-k}}^{\xi_{p+k}} d \xi_{1} \frac{E_{p} E_{1}-\xi_{p} \xi_{1}-\Delta^{2}}{E_{p} E_{1}}\left(\frac{1}{\omega-E_{p}-E_{1}}-\frac{1}{\omega+E_{p}+E_{1}}\right) \\
& \times \frac{1}{\sqrt{\left\{\left[\sqrt{2 m\left(\xi_{p}+\mu\right)}+k\right]^{2}-2 m\left(\xi_{1}+\mu\right)\right\}\left\{2 m\left(\xi_{1}+\mu\right)-\left[\sqrt{2 m\left(\xi_{p}+\mu\right)}-k\right]^{2}\right\}}},
\end{aligned}
$$

where $E_{p}=\sqrt{\xi_{p}^{2}+\Delta^{2}}$ and $E_{1}=\sqrt{\xi_{1}^{2}+\Delta^{2}}$. We should point out some important nuances in the equation above. First, the lower bound of the integral over $\xi_{p}$ is originally $-\mu$. This energy lies deep in the Fermi sea so that we can extend it to negative infinity without incurring significant error.

The factors inside the radical in the last line of Eq. (A8) can be simplified by using the fact that the significant contribution to the integral is from a small interval within the Fermi momentum $p_{F}$ so that

$$
\sqrt{\frac{2\left(\xi_{p}+\mu\right)}{m}} \approx v_{F}
$$

and that we are insterested in small wave vectors $k \approx 0$ so that terms of order $k^{2}$ can be neglected. These approximations give

$$
\begin{aligned}
& \left(\sqrt{2 m\left(\xi_{p}+\mu\right)}+k\right)^{2}-2 m\left(\xi_{p}+\mu\right) \approx 2 m\left(\xi_{p}-\xi_{1}+v_{F} k\right), \\
& 2 m\left(\xi_{p}+\mu\right)-\left(\sqrt{2 m\left(\xi_{p}+\mu\right)}+k\right)^{2} \approx 2 m\left(\xi_{1}-\xi_{p}+v_{F} k\right) .
\end{aligned}
$$

Further we change the integration variable $y \equiv \xi_{1}-\xi_{p}$ and simplify the factor

$$
\frac{E_{p} E_{1}-\xi_{p} \xi_{1}-\Delta^{2}}{E_{1}}=\frac{\sqrt{\xi_{p}^{2}+\Delta^{2}} \cdot \sqrt{\left(\xi_{p}+y\right)^{2}+\Delta^{2}}-\xi_{p}\left(\xi_{p}+y\right)-\Delta^{2}}{\sqrt{\left(\xi_{p}+y\right)^{2}+\Delta^{2}}} .
$$

We are interested in the case when the wavelength is much longer than the size of the Cooper pairs, $v_{F} k \ll \Delta$. Since $|y|<v_{F} k$, we can expand Eq. (A11) in the vicinity of $y \approx 0$ and retain only the leading order. We have

$$
\frac{E_{p} E_{1}-\xi_{p} \xi_{1}-\Delta^{2}}{E_{1}} \approx \frac{\Delta^{2} y^{2}}{2\left(\Delta^{2}+\xi_{p}^{2}\right)^{3 / 2}}
$$

Collecting all these results, we find that Eq. (A8) turns into

$$
\begin{aligned}
P_{k \omega}^{R}= & \frac{m \Delta^{2}}{4 \pi^{2}} \int_{-\infty}^{\infty} d \xi_{p} \int_{-v_{F} k}^{v_{F} k} d y \frac{y^{2}}{\left(\xi_{p}^{2}+\Delta^{2}\right)^{2}} \cdot \frac{1}{\sqrt{\left(v_{F} k-y\right)\left(v_{F} k+y\right)}} \\
& \times\left(\frac{1}{\omega-\sqrt{\xi_{p}^{2}+\Delta^{2}}-\sqrt{\left(\xi_{p}+y\right)^{2}+\Delta^{2}}}-\frac{1}{\omega+\sqrt{\xi_{p}^{2}+\Delta^{2}}+\sqrt{\left(\xi_{p}+y\right)^{2}+\Delta^{2}}}\right) .
\end{aligned}
$$

Using the smallnes of $y$, we can similarly expand the second line in Eq. (A13) as

$$
\frac{1}{\omega \mp \sqrt{\xi_{p}^{2}+\Delta^{2}} \mp \sqrt{\left(\xi_{p}+y\right)^{2}+\Delta^{2}}} \approx \frac{1}{\omega \mp 2 E_{p}} .
$$

Substitution of this expression into Eq. (A13) enables us to evaluate the integral over $y$ by letting $x \equiv y /\left(v_{F} k\right)$ :

$$
\int_{-v_{F} k}^{v_{F} k} \frac{y^{2} d y}{\sqrt{\left(v_{F} k-y\right)\left(v_{F} k+y\right)}}=\left(v_{F} k\right)^{2} \int_{-1}^{1} \frac{x^{2} d x}{\sqrt{(1-x)(1+x)}}=\left(v_{F} k\right)^{2} \frac{\pi}{2} .
$$

Equation (A13) turns into

$$
\begin{aligned}
P_{k \omega}^{R} & =\frac{m \Delta^{2}}{8 \pi}\left(v_{F} k\right)^{2} \int_{-\infty}^{\infty} d \xi_{p} \frac{1}{\left(\xi_{p}^{2}+\Delta^{2}\right)^{2}}\left(\frac{1}{\omega-2 \sqrt{\xi_{p}^{2}+\Delta^{2}}}-\frac{1}{\omega+2 \sqrt{\xi_{p}^{2}+\Delta^{2}}}\right) \\
& =\frac{m \Delta^{2}}{8 \pi}\left(v_{F} k\right)^{2} \int_{-\infty}^{\infty} d \xi_{p} \frac{1}{\left(\xi_{p}^{2}+\Delta^{2}\right)^{3 / 2}} \cdot \frac{1}{\frac{1}{4} \omega^{2}-\Delta^{2}-\xi_{p}^{2}}
\end{aligned}
$$


We denote $\alpha \equiv \sqrt{\frac{1}{4} \omega^{2}-\Delta^{2}}$ and then rewrite $P_{k \omega}^{R}$ as

$$
P_{k \omega}^{R}=\frac{m\left(v_{F} k \Delta\right)^{2}}{8 \pi} \int_{-\infty}^{\infty} d \xi_{p} \frac{1}{\left(\xi_{p}^{2}+\Delta^{2}\right)^{3 / 2}} \cdot \frac{1}{\alpha^{2}-\xi_{p}^{2}} .
$$

Note that the integrand in Eq. (A17) has vanishing contribution for large $\xi_{p}$. In the high frequency regime $\omega \gg 2 \sqrt{2} \Delta$, $\alpha>\Delta$ so that the integral has nigligible contribution for $\xi_{p}>\alpha$. We can then expand

$$
\frac{1}{1-\left(\xi_{p} / a\right)^{2}} \approx \frac{1}{\alpha^{2}}
$$

Putting $\xi_{p}=\Delta \sinh t$ in the integral in Eq. (A17), we find

$$
\int_{-\infty}^{\infty} d \xi_{p} \frac{1}{\left(\xi_{p}^{2}+\Delta^{2}\right)^{3 / 2}}=\frac{1}{\Delta^{2}} \int_{-\infty}^{\infty} \operatorname{sech}^{2} t d t=\left.\frac{1}{\Delta^{2}} \tanh t\right|_{-\infty} ^{\infty}=\frac{2}{\Delta^{2}},
$$

and Eq. (A17) then turns into

$$
P_{k \omega}^{R}=\frac{m v_{F}^{2} k^{2}}{\pi} \cdot \frac{1}{\omega^{2}-4 \Delta^{2}}, \quad \omega \gg 2 \sqrt{2} \Delta
$$

\section{S-wave, Imaginary part}

A similar manipulation can be performed to evaluate the imaginary part of Eq. (A1):

$$
i P_{k \omega}^{I}=\frac{1}{(2 \pi)^{2}} \int d^{2} p \frac{E_{\mathbf{p}} E_{\mathbf{p}+\mathbf{k}}-\xi_{\mathbf{p}} \xi_{\mathbf{p}+\mathbf{k}}-\Delta^{2}}{E_{\mathbf{p}} E_{\mathbf{p}+\mathbf{k}}}\left[-i \pi \delta\left(\omega-E_{\mathbf{p}}-E_{\mathbf{p}+\mathbf{k}}\right)+i \pi \delta\left(\omega+E_{\mathbf{p}}+E_{\mathbf{p}+\mathbf{k}}\right)\right] .
$$

Since $\omega+E_{\mathbf{p}}+E_{\mathbf{p}+\mathbf{k}}>0$, the second Dirac delta function gives zero. We then have

$$
P_{k \omega}^{I}=-\frac{m}{2 \pi} \int_{-v_{F} k}^{v_{F} k} d y \frac{1}{\sqrt{\left(v_{F} k-y\right)\left(v_{F} k+y\right)}} \int_{-\infty}^{+\infty} \frac{d \xi_{\mathbf{p}}}{2 E_{\mathbf{p}}^{2}} \delta\left(\omega-E_{\mathbf{p}}-E_{\mathbf{q}, y}\right),
$$

where $E_{\mathbf{q}, y} \equiv \sqrt{\left(y+\sqrt{E_{p}^{2}-\Delta^{2}}\right)^{2}+\Delta^{2}}$. Note that since $E_{\mathbf{p}}, E_{\mathbf{p}+\mathbf{k}} \geq \Delta$, it follows that $P_{k \omega}^{I}=0$ when $\omega<2 \Delta$. For $\omega \geq 2 \Delta$, we have

$$
P_{k \omega}^{I}=-\frac{m}{8}\left(v_{F} k\right)^{2} \sum_{n} \int_{-\infty}^{\infty} \frac{d \xi_{p}}{E_{p}^{2}} \cdot \frac{\delta\left(\xi_{p}-\xi_{n}\right)}{\left|f^{\prime}\left(\xi_{n}\right)\right|}
$$

where $f\left(\xi_{p}\right) \equiv E_{p}+E_{q}-\omega$ and a prime means derivative with respect to $\xi_{p}$. The sum is over the zeros of $f\left(\xi_{n}\right)=0$. Since $|y|<v_{F} k \ll \Delta$, it follows that $E_{q, y} \approx E_{p}$ which then gives the zeros of $f\left(\xi_{p}\right)$ which are $\xi_{n}= \pm \sqrt{\frac{1}{4} \omega^{2}-\Delta^{2}}$. Finally, we find:

$$
P_{k \omega}^{I}=-\frac{m}{2}\left(v_{F} k\right)^{2} \frac{1}{\sqrt{\omega^{2}-4 \Delta^{2}}} .
$$

\section{P-wave, Real part}

For the p-wave pairing, we can write the gap function as

$$
\Delta_{\mathbf{k}}=f(k)\left(k_{x}-i k_{y}\right)=f(k) k e^{-i \phi},
$$

where $\phi$ is the polar angle measured with respect to the $k_{x}$ axis in k-space. The function $f(k)$ can be found for the concrete p-wave superconductor. Here we keep its general form, which should satisfy the following requirements: (i) $f(k)$ is finite for all $k \in[0, \infty)$ and (ii) $f(k)$ vanishes as $k \rightarrow \infty$. In our case, we take $f(k)$ to be Gaussian :

$$
f(k)=A e^{-\alpha k^{2}},
$$


where $A$ and $\alpha$ are phenomenological parameters. It should be noted, that finding the exact shape of $f(k)$ is unnecessary when we are after the topological properties of the p-wave superconductor only (as long as $f(k)$ satisfies the requirements stated above).

The real part of the polarization can now be written as

$$
\begin{aligned}
P_{k \omega}^{R}= & \frac{1}{(2 \pi)^{2}} \int d^{2} p\left[\left(1-\frac{\xi_{\mathbf{p}} \xi_{\mathbf{p}+\mathbf{k}}}{E_{\mathbf{p}} E_{\mathbf{p}+\mathbf{k}}}-\frac{\mathcal{R} e\left\{\Delta_{\mathbf{p}}^{*} \Delta_{\mathbf{p}+\mathbf{k}}\right\}}{E_{\mathbf{p}} E_{\mathbf{p}+\mathbf{k}}}\right)\left(\frac{1}{\omega-E_{\mathbf{p}}-E_{\mathbf{p}+\mathbf{k}}}-\frac{1}{\omega+E_{\mathbf{p}}+E_{\mathbf{p}+\mathbf{k}}}\right)\right. \\
& \left.+\pi \frac{\mathcal{I} m\left\{\Delta_{\mathbf{p}}^{*} \Delta_{\mathbf{p}+\mathbf{k}}\right\}}{E_{\mathbf{p}} E_{\mathbf{p}+\mathbf{k}}}\left(\delta\left(\omega+E_{\mathbf{p}}+E_{\mathbf{p}+\mathbf{k}}\right)-\delta\left(\omega-E_{\mathbf{p}}-E_{\mathbf{p}-\mathbf{k}}\right)\right)\right] .
\end{aligned}
$$

At the quantum critical point $\mu=0$, the argument of the Dirac delta function $\delta\left(\omega+E_{\mathbf{p}}+E_{\mathbf{p}+\mathbf{k}}\right)$ vanishes at $\mathbf{k}=0$ and $\omega=0$. Since we are not interested in this critical case for now, we take $\mu \neq 0$ for which $\omega+E_{\mathbf{p}}+E_{\mathbf{p}+\mathbf{k}}>0$ and the mentioned Dirac delta is dropped.

Furthermore we evaluate analytically the term involving the other Dirac delta. First we rewrite

$$
\Delta_{\mathbf{p}+\mathbf{k}}=f(|\mathbf{p}+\mathbf{k}|)|\mathbf{p}+\mathbf{k}| e^{-i \theta} .
$$

Without loss of generality, we can choose $\mathbf{k}$ to lie along the $p_{x}$ axis. We then find

$$
\theta=\sin ^{-1}(p \sin \phi)
$$

where $\phi$ is the polar angle and it is simultaneously the angle between $\mathbf{k}$ and $\mathbf{p}$.

Following a similar procedure as in Section A (s-wave case), we put $p_{1} \equiv|\mathbf{p}+\mathbf{k}|$ so that the Dirac delta term in Eq. A27) becomes

$$
\begin{aligned}
P_{k \omega}^{\prime R} & =-\frac{1}{4 \pi} \int d^{2} p \frac{\mathcal{I} m\left\{\Delta_{\mathbf{p}}^{*} \Delta_{\mathbf{p}+\mathbf{k}}\right\}}{E_{\mathbf{p}} E_{\mathbf{p}+\mathbf{k}}} \delta\left(\omega-E_{\mathbf{p}}-E_{\mathbf{p}-\mathbf{k}}\right) \\
& =-\frac{1}{2 \pi} \int_{0}^{\infty} \int_{p-k}^{p+k} d p_{1} \frac{f(p) f\left(p_{1}\right) p_{1}^{2}}{k E_{\mathbf{p}} E_{\mathbf{p}+\mathbf{k}}} \cos \theta \delta\left(\omega-E_{\mathbf{p}}-E_{\mathbf{p}-\mathbf{k}}\right),
\end{aligned}
$$

where

$$
\theta=\sin ^{-1}\left[\frac{1}{2 p_{1} k} \sqrt{4 p^{2} k^{2}-\left(p_{1}^{2}-p^{2}-k^{2}\right)^{2}}\right] .
$$

Then we perform another change of integration variables:

$$
\begin{aligned}
\xi_{p} & =\frac{p^{2}}{2 m}-\mu, \\
\xi_{p_{1}} & =\frac{p_{1}^{2}}{2 m}-\mu,
\end{aligned}
$$

so that Eq. (A30) now becomes

$$
P_{k \omega}^{\prime R}=-\frac{m \sqrt{2 m}}{2 \pi k v_{F}} \int_{-\infty}^{\infty} d \xi_{p} \int_{\xi_{p-k}}^{\xi_{p+k}} d \xi_{1} \frac{\sqrt{\xi_{1}+\mu}}{E_{p} E_{1}} f\left(\xi_{p}\right) f\left(\xi_{1}\right) \cos \theta \delta\left(\omega-E_{p}-E_{1}\right)
$$

where $f(p)=A \exp \left\{-2 m \alpha\left(\xi_{p}+\mu\right)\right\}$ and $E_{p}=\sqrt{\xi_{p}^{2}+f^{2}(p)}$. The upper and lower limits of the $\xi_{1}$ integration can be rewritten as

$$
\begin{aligned}
\xi_{p \pm k} & =\frac{(p \pm k)^{2}}{2 m}-\mu \\
& =\xi_{p} \pm v_{F} k+\frac{k^{2}}{2 m} .
\end{aligned}
$$

The relevant contribution from the $\xi_{p}$ integration comes from a close neighborhood around the Fermi surface, so that $\xi_{p}$ in Eq. (A9) we can approximate as $\xi_{p} \approx \frac{1}{2} m v_{F}^{2}-\mu$. Further, since we are interested in small $k$, we can drop the $k^{2}$ term. The $\xi_{p}$ and $\xi_{1}$ integrations in eq.(A33) can now be interchanged and the former integral can be evaluated to get

$$
P_{k \omega}^{\prime R}=-\frac{m \sqrt{2 m}}{2 \pi k v_{F}} \sum_{n= \pm} \int_{-v_{F} k}^{v_{F} k} d \xi_{1} \frac{f\left(\xi_{n}\right) f\left(\xi_{1}\right) \sqrt{\xi_{1}+\mu}}{E_{1}\left|g^{\prime}\left(\xi_{n}\right)\right| \sqrt{\xi_{n}^{2}+f_{0}^{2}}} \cos \theta
$$



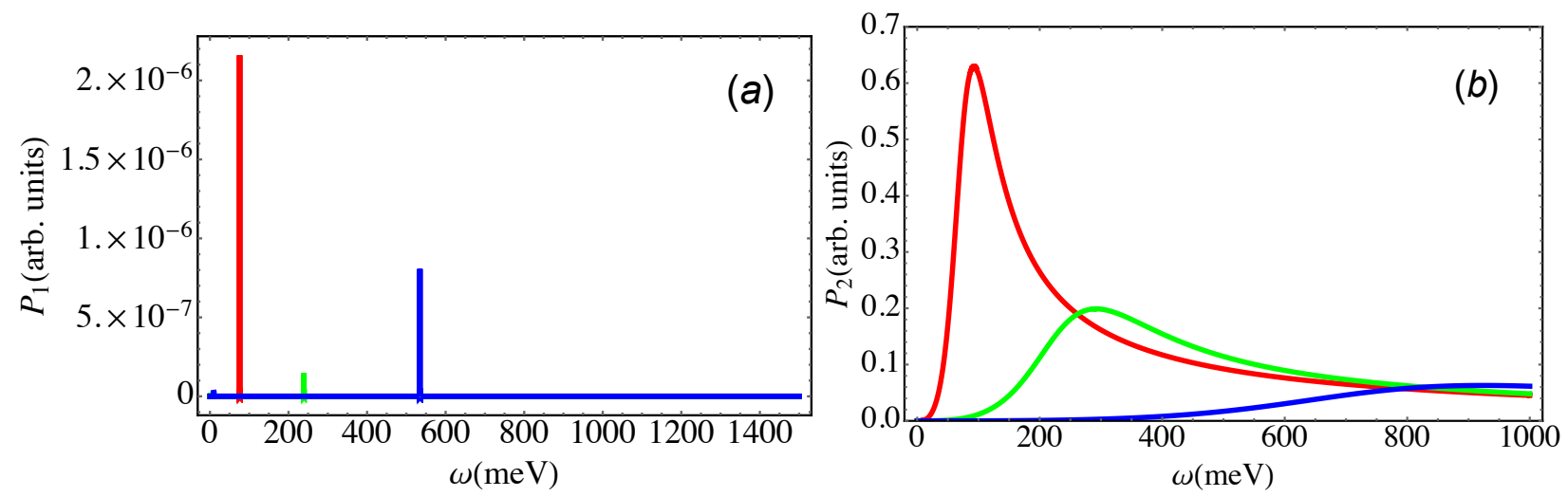

FIG. 4. Power absorption spectrum when the interlayer interaction is turned off (a) Current is monitored in the 2DEG. (b) Current is monitored in the superconductor.

where $g\left(\xi_{p}\right)=E_{p}+E_{1}-\omega$ and the prime over $g\left(\xi_{p}\right)$ denotes derivative with respect to $\xi_{p}$. The roots of $g\left(\xi_{p}\right)=0$ are

$$
\xi_{ \pm}=-m f_{0}^{2} \pm \frac{1}{2} \sqrt{4 m^{2} f_{0}^{4}-4\left[2 m \mu f_{0}^{2}-\left(\omega-E_{1}\right)^{2}\right]} .
$$

This last integral in (A35) can be easily evaluated numerically.

\section{P-wave, Imaginary part}

The imaginary part of the polarization operator reads

$$
\begin{aligned}
P_{k \omega}^{I}= & \frac{1}{(2 \pi)^{2}} \int d^{2} p\left[\pi\left(1-\frac{\xi_{\mathbf{p}} \xi_{\mathbf{p}+\mathbf{k}}}{E_{\mathbf{p}} E_{\mathbf{p}+\mathbf{k}}}-\frac{\mathcal{R} e\left\{\Delta_{\mathbf{p}}^{*} \Delta_{\mathbf{p}+\mathbf{k}}\right\}}{E_{\mathbf{p}} E_{\mathbf{p}+\mathbf{k}}}\right)\left(\delta\left(\omega+E_{\mathbf{p}}+E_{\mathbf{p}+\mathbf{k}}\right)-\delta\left(\omega+E_{\mathbf{p}}-E_{\mathbf{p}-\mathbf{k}}\right)\right)\right. \\
& \left.-\frac{\mathcal{I} m\left\{\Delta_{\mathbf{p}}^{*} \Delta_{\mathbf{p}+\mathbf{k}}\right\}}{E_{\mathbf{p}} E_{\mathbf{p}+\mathbf{k}}}\left(\frac{1}{\omega-E_{\mathbf{p}}-E_{\mathbf{p}+\mathbf{k}}}-\frac{1}{\omega+E_{\mathbf{p}}+E_{\mathbf{p}+\mathbf{k}}}\right)\right] .
\end{aligned}
$$

Similar steps (as in Subsection C above) can be followed to find

$$
\begin{aligned}
P_{k \omega}^{I}= & -\frac{m}{2 \pi} \sum_{n= \pm} \int_{-v_{F} k}^{v_{F} k} d \xi_{1} \frac{1}{\left|g^{\prime}\left(\xi_{n}\right)\right| \sqrt{\left(\xi_{n}-\xi_{1}+v_{F} k\right)\left(\xi_{1}-\xi_{n}+v_{F} k\right)}} \\
& \times\left(1-\frac{\xi_{n} \xi_{1}}{E_{n} E_{1}}-\frac{\sqrt{m\left(\xi_{1}+\mu\right)}}{2 \sqrt{2} k E_{n} E_{1}} f\left(\xi_{n}\right) f\left(\xi_{1}\right)\left[2 m\left(\xi_{1}-\xi_{n}\right)-k^{2}\right] \cos \theta\right) .
\end{aligned}
$$

\section{Appendix B: The external potential $W_{k \omega}$}

In terms of vector and scalar potentials, a time-dependent electric field is given by

$$
\mathbf{E}(\mathbf{r}, t)=-\frac{\partial \mathbf{A}}{\partial t}-\nabla \phi
$$

Since the incident electric field is parallel to the plane of the electron gas sample at $z=0$ and the tangential component of the electric field obeys the boundary condition $\lim _{z \rightarrow 0^{+}} E_{||}=\lim _{z \rightarrow 0^{-}} E_{\|}$, we can neglect the effect of free charges on this field. The Lorenz gauge then gives the wave equations

$$
\begin{aligned}
\nabla^{2} \phi-\frac{\partial^{2} \phi}{\partial t^{2}} & =0, \\
\nabla^{2} \mathbf{A}-\frac{\partial^{2} \mathbf{A}}{\partial t^{2}} & =0,
\end{aligned}
$$




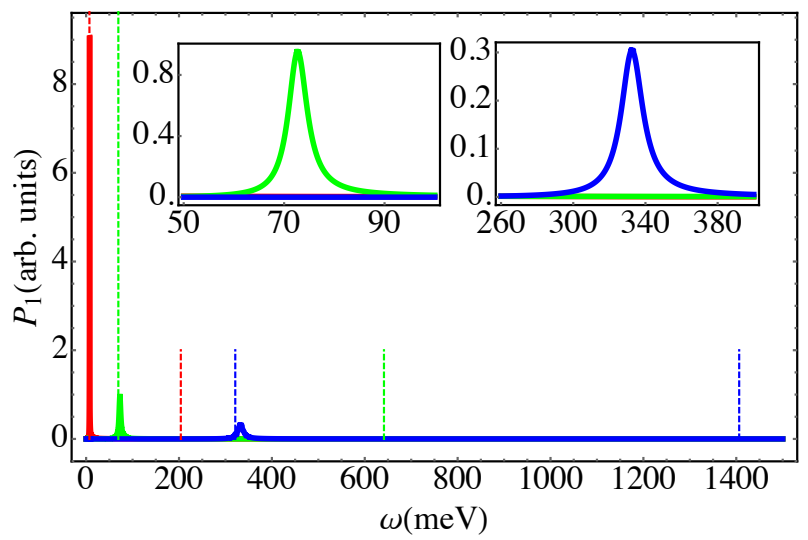

FIG. 5. Power absorption as a function of $\omega$ when there is no external EM field on the superconductor layer for $k=1.0$ (red), $5.0 \times 10^{1}$ (green), $1.0 \times 10^{2} \mathrm{meV}$ (blue). Inset: zoom-in for $50 \leq \omega<95 \mathrm{meV}$ and $260 \leq \omega<390$.

where $\nabla^{2}$ is the laplacian in three-dimensional space $\mathbf{R}$. It can be verified that

$$
\begin{aligned}
\phi(\mathbf{R}, t) & =-x E_{0} e^{-i(q z+\omega t)} \\
\mathbf{A}(\mathbf{R}, t) & =\frac{q x}{\omega} E_{0} e^{-i(q z+\omega t)} \hat{\mathbf{k}}
\end{aligned}
$$

obey the wave equations (B2) with $\omega=q$.

Note that Eq. (27) is similar to the electrostatic case with uniform electric field along the $\mathrm{x}$ axis apart from the plane wave factor. Hence, we can still use $W_{k \omega}=\frac{e E_{0}}{i k}$ analogous to the electrostatic case.

\section{Appendix C: Photoabsorption spectrum in case if the interlayer coupling is switched off}

Figure 4 shows the power spectrum when the coupling between the layers is switched off. The left hand side panel shows the case when the current is monitored in the 2DEG layer. It reveals a sharp peak contribution from the lower modes. This is expected since when there is no interlayer coupling, we have $P_{1}(\omega) \propto \Pi_{k \omega}^{I}=0$ for $\omega>2 \Delta$. The sharp peaks occur at the lower hybrid modes where the denominator in the formula for $P_{1}(\omega)$ gives zero. Note the disappearance of three right-most peaks (compare with Fig. 3 in the main text). This confirms that the three broad right-most peaks in Fig. $3 \mathrm{~b}$ are primarily due to the superconductor.

The right hand side panel similarly shows the case when the current is monitored in the superconductor layer. It shows that the three left-most peaks disappear which confirms that they are primarily due to the 2DEG layer.

\section{Appendix D: EMF is exposed to the 2DEG only}

We now investigate the power absorption in the normal layer when the external EM field in the superconductor is turned off by setting $g_{N}=1$ and $g_{S}=0$ in Eq. (8), see Fig. 5. It shows that the contribution of the upper hybrid modes decreases as compared to Fig. 3 in the main text and the curves in the vicinity of the upper hybrid modes are quite broad. The opposite is true for the lower hybrid modes: their contribution is significantly enhanced, as expected, since the superconductor is not exposed to light. 